\title{
The Static and Stability Out-of-Plane Analysis of 2-D Curved Beam with Variable Curvatures*
}

\author{
Minghan LIN**, Huiching WANG** and Kuanchung LIN** \\ ${ }^{* *}$ Department of Applied Mathematics, National Chung Hsing University, Taiwan \\ 250 KuoKuang Rd., Taichung 402, Taiwan \\ E-mail: hcwang@amath.nchu.edu.tw
}

\begin{abstract}
The out of plane static analysis and stability analysis of curved beams of variable curvature on 2-D plane are investigated. The general solutions under various loadings are demonstrated. The analytical solutions of circular, cycloid, and spiral curved beams under pure moments are shown. The analytical solutions of circle, cycloid logarithmic spiral and spiral cantilever curved beams under concentrated load are presented as well. The out-of-plane critical load of a circular ring under uniform radial load is derived. Several critical loads are derived for various out-of-plane boundary conditions. The results are compared to the result by ANSYS. The results are consistent.
\end{abstract}

Key words: Out of Plane, Curved Beam, Stability, Analytical Solution

\section{Introduction}

There are several approaches to the analysis of out-of-plane deformation of straight beams or curved beams on a 2-D plane. One way is to model the beam as a beam in 3-dimensional space. Green [1] treated the rod as a three dimensional elastic body. Based on the power series expansion to a convected coordinate, the approximate 3-D model was derived. Green and Naghdi [2], Naghdi [3], Green [4] applied one dimensional director theory to model a rod in 3-D. The rod is treated as a curve. Green [5] showed some relationship between two approaches. Those two ways can derive approximate models for 2-D curved beams. However the system equations are very complicated. Yu [6] presented an analytical method for a curved twisted beam. A curvilinear coordinate system was attached along the centroid line. An analytical form of static general solution was derived.

One other approach is isolating the out of plane equations. The quantities of in plane forces, moment, deformations are all decoupled. In this way, the system equations become simple. Malekzadeh [7] applied DMQ method to analyze the static circular arch under various boundary conditions. The effect of shear deformation was included. Kang [8] used both exact solution and DMQ method to the out-of-plane static analysis of a circular beam under a torque at the end pint. Neglecting the shear deformation, exact solutions can be obtained. The numerical technique DMQ method was applied based on power series expansion. The study was restricted to circular curved beam.

The analytical solutions of dynamic analysis are very limited. Yilditrim [9] applied transfer matrix method to study symmetric circular composite bar. Six natural frequencies were found. Out-of-plane dynamic noncircular curved beams were studied by numerical Laplace transform method (Huang [10]). The shear deformation, rotary inertia and damping effect were all included.

[DOI: 10.1299/jmmp.6.886]

Copyright (C) 2012 by JSME 
One other topic is out-of-plane stability analysis. In the stability analysis, the in-plane and out-of-plane system equations are coupled. Rhodes [11] studied an orthotropic thin walled structure by a semi-analytical approach. The analysis combined part of plate and beam theories involving the out-of-plane deformation and in-plane deformation. The out-of-plane buckling of a nonlinear and orthotropic concrete wall was derived by FEM (Attard [12]). An element of 16 degree of freedom of plate bending element was derived for this wall. By using principle of minimum potential energy, the out-of-plane elastic stability theory was developed for thin wall circular arches (Lim [13]). Closed form solutions were obtained for uniformed distributed moment and uniformly distributed radial load. Closed form solutions of the torsional buckling were obtained as well.

In this research, the out-of-plane static and stability deformation of curved beams of variable curvature on 2-D plane will be studied. Only analytical solutions are interested in this study. The radius of curvature and angle of tangent slope (Lin [14]) are used to describe the curved beams. In the static analysis, the out-of-plane system equations are decoupled to in-plane system equations. The static general solutions will be derived. Some analytical solutions are demonstrated. In the stability analysis, the in-plane and out-of-plane system equations are coupled together. The analytical solution of the critical load of a circular ring under uniform radial pressure is demonstrated. The results also compared to the results by ANSYS. The results are consistent.

\section{General Description}

Consider a curved beam (Fig.1) of variable curvature on $x-y$ plane. The curve beam is under either loads out of $x-y$ plane or loads on $x-y$ plane or both. In this research, the out of plane deformation will be investigated. This out of plane deformation may be caused by the loading on $x-y$ plane. It yields to stability analysis. The out of plane deformation may also be caused by the loading along $z$-direction.

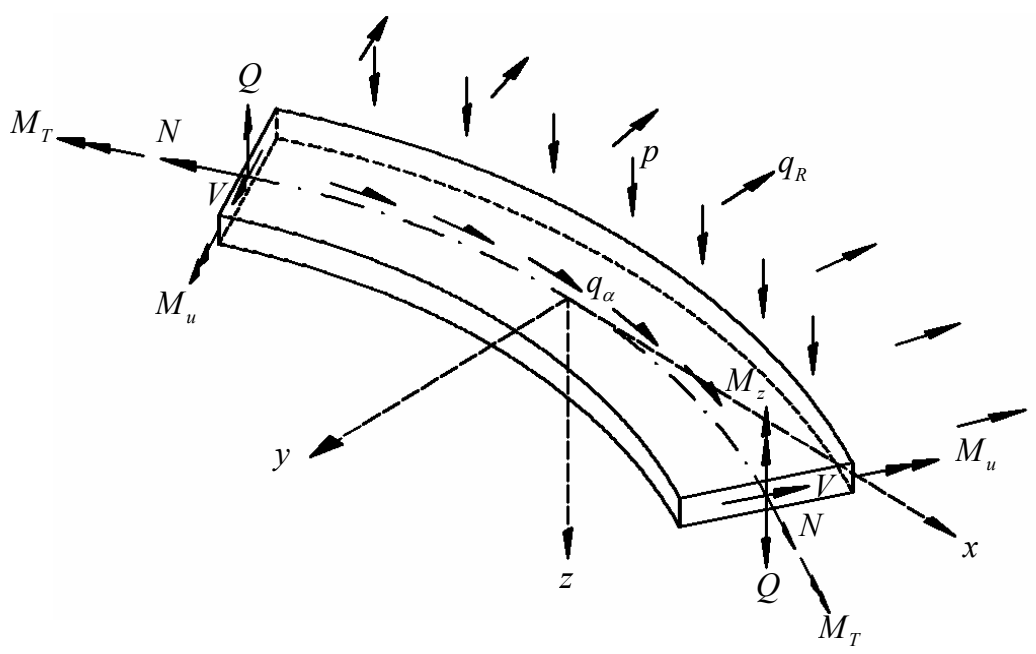

Fig.1 Sign convention of forces and moments of a 2-D curved beam

The sign convention of forces and moments are also shown. The torque $M_{T}$ is along axial direction and the moment $M_{u}$ is along radial direction. These two moments will cause out of plane rotation of cross section. The shear force $Q$ is along $z$-direction. The axial tension $N$ along axial direction and shear force $V$ along radial direction are two components of in plane forces. These two components may cause in plane deformation in static analysis. They may also cause out of plane deformation in stability analysis. The in plane moment $M_{z}$ along $z$ direction may cause the in plane rotation. The schematic drawing of components of deformation is shown in Fig. 2. 


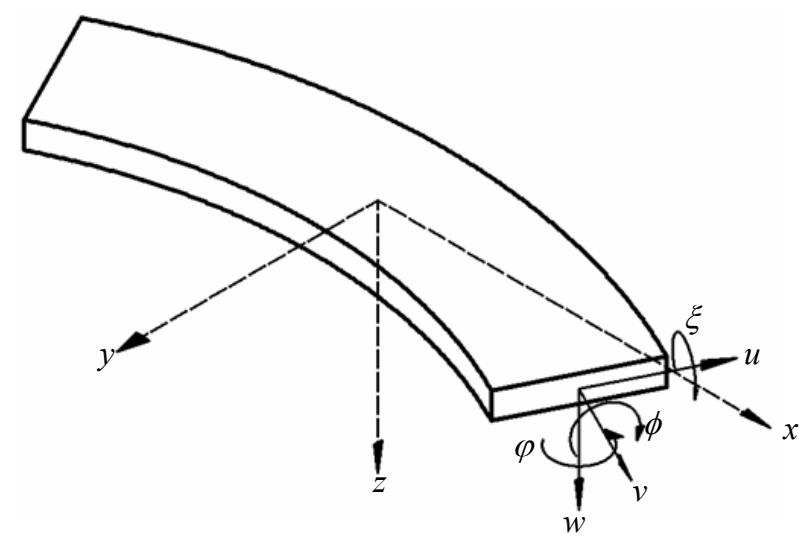

Fig.2 Sign convention of displacement components

To show the analysis, the variables $R, x, y, s, u, v, w$ are nondimensionalized with respect to a referential radius $R_{0}$, forces $N, V, q_{\alpha}, q_{R}$ are respect to $E A, M_{u}, M_{T}$ are respect to $E I / R_{0}$, respectively. The displacement $w$ is along $z$-direction, the rotation deformation $\xi$ is along radial direction. The definition of the variable $\varphi$ is rotation angle along $z$ direction and the twisted angle $\phi$ is along axial direction. Here the shear deformation is ignored, i.e.

$$
\xi=\frac{d w}{d s},
$$

where $d s$ is the arc length element. The equilibrium equations of $x-y$ plane components can be evaluated by force balance, i.e.

$$
\frac{d N}{d s}+\frac{V}{R}=-q_{\alpha}, \quad-\frac{N}{R}+\frac{d V}{d s}=-q_{R}, \quad \frac{d M_{z}}{d s}=V .
$$

Eq.(2a) is the balance of axial component of force. Eq.(2b) is the balance of tangential component of force and Eq.(2c) is the balance of moment along z-direction. The equilibrium of out of plane components due to balance are

$$
\frac{d M_{T}}{d s}-\frac{M_{u}}{R}-V \xi=0, \quad \frac{M_{T}}{R}+\frac{d M_{u}}{d s}-N \xi=Q, \quad \frac{d Q}{d s}=-p,
$$

where Eqs.(3a, 3b) are the balance of moments along axial direction and radial direction respectively. Eqs.(3c) is the balance of shear force along $z$-direction.

In Eq.(3), the out of plane moment caused by in plane axial force $N$ and shear force $V$ are included. It will yield the effect of stability. In the case of static analysis, it will be neglected. The in plane forces and displacements relationship are

$$
N=\frac{d v}{d s}+\frac{u}{R}, \quad M_{z}=\frac{d \varphi}{d s}, \quad \varphi=\frac{v}{R}-\frac{d u}{d s},
$$

where $v, u, \varphi$ are the deformation along tangential direction, the deformation along radial direction and the rotation angle on $x-y$ plane, respectively. The differential equations are decouple to out of plane deformation and force component. Eqs. $(2,4)$ form a system of differential equations of variables of $N, V, M_{z}, u, v, \varphi$. Here only the cases that cause out of plane deformation will be demonstrated. The forces and displacements relationship of out of plane are

$$
M_{u}=\frac{d \xi}{d s}+\frac{\phi}{R}, \quad M_{T}=\frac{1}{Z}\left(\frac{d \phi}{d s}-\frac{\xi}{R}\right), \quad \xi=\frac{d w}{d s},
$$

where $Z$ is a dimensionless constant defined by

$$
Z=\frac{E I}{C}
$$

which is the ratio of rigidity of moment $E I$ to rigidity of torque $C$. 
Eqs. $(3,5)$ form a system of six differential equations of variables $M_{u}, M_{T}, Q$ and $\xi, \phi, w$. The in plane forces of $N, V$ are coupled in these equations. The complete system of twelve differential Eqs. $(2,3,4,5)$ involve with variables of $N, V, M_{z}, u, v, \varphi$ and $M_{u}, M_{T}, Q, \xi, \phi, w$. Since the system of in plane Eqs. $(2,4)$ are decoupled from the out of plane Eqs. $(3,5)$, Eqs. $(2,4)$ can be solved independently. The general solutions of Eqs. $(2,4)$ with suitable boundary conditions have a standard form (Lin[14]). The solutions are expressed in terms of fundamental geometry quantities such as first moment of arc length with respect to $x, y$ axes, or second moments of arc length.

The difficulties arise in Eqs.(3). Once $R$ is assigned and $N, V$ are solved. Again as in the plane stability studies, Mathieu type differential equations appear. The solutions are involved special functions. The analytical solutions in the form of fundamental functions are limited.

This research provides the general solutions of some curved beams. Some curves such as circle, cycloid, logarithmic spiral, and spiral are derived in this study. The focus will be on out-of-plane deformation.

\section{Static out of plane analysis}

\subsection{General solutions}

Excluding the effect of stability, the in plane forces $N, V$ have no effect to the out of plane moments. Therefore $N, V$ are decoupled to the out-of-plane analysis. Eq.(3) then can be rewritten as

$$
\frac{d M_{T}}{d s}-\frac{M_{u}}{R}=0, \quad \frac{M_{T}}{R}+\frac{d M_{u}}{d s}=Q, \quad \frac{d Q}{d s}=-p .
$$

Change variable by using

$$
d s=R d \alpha
$$

where $\alpha$ is the tangent angle at any point. Here radius of curvature $R$ is expressed as a function of $\alpha$. Hence Eq.(7c) can be solved as

$$
Q=-\int_{0}^{\alpha} p R d \alpha+Q_{0}
$$

Here it is chosen that at $\alpha=0, x=0 y=0$. The coordinates of $x, y$ can be evaluated by

$$
x=\int_{0}^{\alpha} R \cos \bar{\alpha} d \bar{\alpha}, \quad y=\int_{0}^{\alpha} R \sin \bar{\alpha} d \bar{\alpha} .
$$

Using Eq.(8), Eqs.(7a,b) can be expressed by a set of differential equations in terms of tangent angle $\alpha$. Substituting Eq.(7b) into Eq.(7a), the analytical solution of $M_{T}$ can be obtained as

$$
M_{T}=A_{1} \cos \alpha+A_{2} \sin \alpha-\cos \alpha \int_{0}^{\alpha} Q R \sin \alpha d \alpha+\sin \alpha \int_{0}^{\alpha} Q R \cos \alpha d \alpha .
$$

Taking derivative of Eq.(7a), the moment $M_{u}$ can be expressed as

$$
M_{u}=-A_{1} \sin \alpha+A_{2} \cos \alpha+\sin \alpha \int_{0}^{\alpha} Q R \sin \alpha d \alpha+\cos \alpha \int_{0}^{\alpha} Q R \cos \alpha d \alpha .
$$

In Eqs.(11,12), $A_{1}, A_{2}$ are two constants which can be obtained by suitable boundary conditions. $A_{1}$ denotes the reaction torque at $\alpha=0$, and $A_{2}$ denotes the reaction moment at $\alpha=0$.

To solve the displacement field, changing the variable from $s$ to $\alpha$, the nondimensional Eq.(5) can be written as

$$
\frac{d \xi}{d \alpha}+\phi=M_{u} R, \quad-\xi+\frac{d \phi}{d \alpha}=Z M_{T} R, \quad \frac{d w}{d \alpha}=\xi R
$$

By taking derivative of Eq. (13b) and combining with Eq. (13a), Eqs.(13a, b) can be rearranged as

$$
\frac{d^{2} \phi}{d \alpha^{2}}+\phi=M_{u} R+Z \frac{d}{d \alpha}\left(M_{T} R\right)
$$


Since $M_{u}$ and $M_{T}$ can be obtained from Eqs.(11,12), the solution of $\phi$ can be expressed as

$$
\begin{aligned}
\phi & =A_{3} \cos \alpha+A_{4} \sin \alpha-\cos \alpha \int_{0}^{\alpha} M_{u} R \sin \bar{\alpha} d \bar{\alpha}+\sin \alpha \int_{0}^{\alpha} M_{u} R \cos \bar{\alpha} d \bar{\alpha} \\
& +Z \cos \alpha \int_{0}^{\alpha} M_{T} R \cos \bar{\alpha} d \bar{\alpha}+Z \sin \alpha \int_{0}^{\alpha} M_{T} R \sin \bar{\alpha} d \bar{\alpha},
\end{aligned}
$$

where $A_{3}, A_{4}$ are two constants determined by suitable displacement boundary conditions. Substituting Eq.(15) into Eq.(13b), taking derivative of $\phi$, the rotation angle $\xi$ can be obtained

$$
\begin{aligned}
\xi & =-A_{3} \sin \alpha+A_{4} \cos \alpha+\sin \alpha \int_{0}^{\alpha} M_{u} R \sin \bar{\alpha} d \bar{\alpha}+\cos \alpha \int_{0}^{\alpha} M_{u} R \cos \bar{\alpha} d \bar{\alpha} \\
& -Z \sin \alpha \int_{0}^{\alpha} M_{T} R \cos \bar{\alpha} d \bar{\alpha}+Z \cos \alpha \int_{0}^{\alpha} M_{T} R \sin \bar{\alpha} d \bar{\alpha}
\end{aligned}
$$

The displacement $w$ is then integrated once to

$$
w=\int_{0}^{\alpha} \xi R d \bar{\alpha}+w_{0},
$$

where $w_{0}$ is a constant denotes the displacement at $\alpha=0$. Hence the solutions of $Q, M_{T}, M_{u}, \phi$, $\xi, w$ are Eqs.(9), (11,12), (15,16,17) with $Q_{0}, A_{1}, A_{2}, A_{3}, A_{4}, w_{0}$ six constants. These constants are determined by suitable boundary conditions. In general, three forces boundary conditions and three displacement boundary conditions are needed. Once the radius of curvature is given, the integrals can be carried out. It is interesting to point out a simple case

$$
p=0 \text {. }
$$

In other words, there is no distributed load. Eq.(9) yields

$$
Q=Q_{0}
$$

With the help of Eq.(19), Eq.(11) yields

$$
M_{T}=A_{1} \cos \alpha+A_{2} \sin \alpha+Q_{0}(x \sin \alpha-y \cos \alpha),
$$

and Eq.(12) yields

$$
M_{u}=-A_{1} \sin \alpha+A_{2} \cos \alpha+Q_{0}(x \cos \alpha+y \sin \alpha) .
$$

Hence the force reactions are expressed in terms of $x, y$ coordinates and tangent angle $\alpha$. Substituting Eqs.(20), (21) into Eq.(15), the twisted angle $\phi$ and rotation angle $\xi$ can be expressed as

$$
\begin{aligned}
\phi & =A_{3} \cos \alpha+A_{4} \sin \alpha-\cos \alpha\left(A_{1} R_{s s}-A_{2} R_{s c}\right)+\sin \alpha\left(A_{1} R_{s c}-A_{2} R_{c c}\right) \\
& +Z \cos \alpha\left(A_{1} R_{c c}+A_{2} R_{s c}\right)+Z \sin \alpha\left(A_{1} R_{s c}+A_{2} R_{s s}\right) \\
& +Q_{0}\left[\cos \alpha\left(x_{s c}+y_{s s}\right)-\sin \alpha\left(x_{c c}+y_{s c}\right)\right]+Z Q_{0}\left[\cos \alpha\left(x_{s c}-y_{c c}\right)+\sin \alpha\left(x_{s s}-y_{s c}\right)\right], \\
\xi & =-A_{3} \sin \alpha+A_{4} \cos \alpha+\sin \alpha\left(A_{1} R_{s s}-A_{2} R_{s c}\right)+\cos \alpha\left(A_{1} R_{s c}-A_{2} R_{c c}\right) \\
& -Z \sin \alpha\left(A_{1} R_{c c}+A_{2} R_{s c}\right)+Z \cos \alpha\left(A_{1} R_{s c}+A_{2} R_{s s}\right) \\
& +Q_{0}\left[-\sin \alpha\left(x_{s c}+y_{s s}\right)-\cos \alpha\left(x_{c c}+y_{s c}\right)\right]+Z Q_{0}\left[-\sin \alpha\left(x_{s c}-y_{c c}\right)+\cos \alpha\left(x_{s s}-y_{s c}\right)\right],
\end{aligned}
$$

where $R_{s c}, R_{s s}, R_{c c}$ are defined as

$$
R_{s c}=\int_{0}^{\alpha} R(\alpha) \sin \alpha \cos \alpha d \alpha, \quad R_{s s}=\int_{0}^{\alpha} R(\alpha) \sin ^{2} \alpha d \alpha, \quad R_{c c}=\int_{0}^{\alpha} R(\alpha) \cos ^{2} \alpha d \alpha,
$$

which denote the moments of arc length with respect to $x, y$ coordinates. The others $x_{s c}, x_{s s}$, $x_{c c}, y_{s c}, y_{s s}, y_{c c}$ are defined as 


$$
\begin{aligned}
& x_{s c}=\int_{0}^{\alpha} x(\bar{\alpha}) \sin \bar{\alpha} \cos \bar{\alpha} d \bar{\alpha}, \quad x_{s s}=\int_{0}^{\alpha} x(\bar{\alpha}) \sin ^{2} \bar{\alpha} d \bar{\alpha}, \quad x_{c c}=\int_{0}^{\alpha} x(\bar{\alpha}) \cos ^{2} \bar{\alpha} d \bar{\alpha}, \\
& y_{s c}=\int_{0}^{\alpha} y(\bar{\alpha}) \sin \bar{\alpha} \cos \bar{\alpha} d \bar{\alpha}, \quad y_{s s}=\int_{0}^{\alpha} y(\bar{\alpha}) \sin ^{2} \bar{\alpha} d \bar{\alpha}, \quad y_{c c}=\int_{0}^{\alpha} y(\bar{\alpha}) \cos ^{2} \bar{\alpha} d \bar{\alpha},
\end{aligned}
$$

which denote the second moments of length with respect to $x, y$ coordinates. It is the main difference between straight beam and curved beam. In straight beam analysis, the only required quantities are the area and moment of inertia of the cross section. Since the straight beam is always lied on the axis or one direction, there is no need to evaluate the components of length with respect to $x$ or $y$ axis. For a curved beam, the curved element is always laid on $x-y$ plane. It is needed to evaluate the components with respect to $x, y$ axes.

\subsection{Cantilever curved beam under a pure torque}

A cantilever beam starting from $\alpha=0$ to $\alpha=\pi / 2$ is considered (Fig.3).

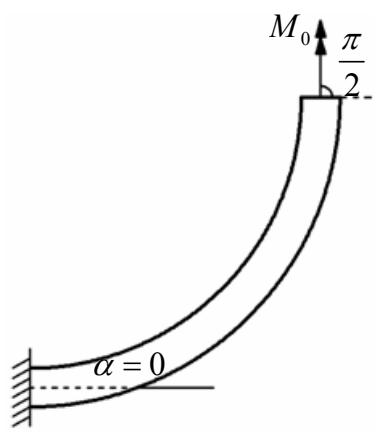

Fig.3 A cantilever curved beam under a concentrated torque $M_{0}$

A concentrated torque $M_{0}$ is applied at the free end $\alpha=\pi / 2$. At $\alpha=0$, it is fixed. At fixed end $\alpha=0$, there is no rotation and deflection, i.e. $\phi(0)=0, \xi(0)=0, w(0)=0$. From Eqs. $(15,16,17)$, the constants are

$$
A_{3}=A_{4}=w_{0}=0 \text {. }
$$

The constants are easily solved due to the geometrical arrangement. At the free end, since there is no distributed load and shear force, the resultant shear $Q$ is

$$
Q=0 .
$$

Hence the resultant moments can be simplified to

$$
M_{T}=A_{1} \cos \alpha+A_{2} \sin \alpha, \quad M_{u}=-A_{1} \sin \alpha+A_{2} \cos \alpha .
$$

At the free end $\alpha=\pi / 2$, the moment conditions $M_{u}(\pi / 2)=0, M_{T}(\pi / 2)=M_{0}$ imply

$$
M_{T}=M_{0} \sin \alpha, \quad M_{u}=M_{0} \cos \alpha .
$$

Substituting Eqs.(27) into Eqs.(15,16), the solutions of rotation angles $\phi, \xi$ are

$$
\begin{aligned}
& \phi=M_{0}\left\{\cos \alpha R_{s c}-\sin \alpha R_{c c}+Z\left(\cos \alpha R_{s c}+\sin \alpha R_{s s}\right)\right\}, \\
& \xi=M_{0}\left\{-\sin \alpha R_{s c}-\cos \alpha R_{c c}+Z\left(-\sin \alpha R_{s c}+\cos \alpha R_{s s}\right)\right\} .
\end{aligned}
$$

The deflection $w$ can be written with the help of Eq.(17)

$$
w=M_{0} \int_{0}^{\alpha}\left\{-\sin \alpha R_{s c}-\cos \alpha R_{c c}+Z\left(-\sin \alpha R_{s c}+\cos \alpha R_{s s}\right)\right\} R d \alpha .
$$

There is no simple form to express the deflection $w$. Once the radius of curvature is given, the geometric quantities $R_{s c}, R_{c c}, R_{s s}$ can be evaluated in terms of $\alpha$. Hence all the deformation $\xi, \phi, w$ are all expressed in terms of $\alpha$. Some analytical solutions of various curves such as circle, cycloid, logarithmic spiral, and spiral are listed in Table 1. 
Table 1 Dimensionless deflections of various cantilever beams under a concentrated torque $M_{0}$ at free end

\begin{tabular}{|c|c|c|}
\hline Curve Type & $R$ & $\xi, \phi, w$ \\
\hline Circle & 1 & $\begin{array}{l}\xi=\frac{1}{2} M_{0}[\alpha \cos \alpha(1+Z)+\sin \alpha(1-Z)] \\
\phi=\frac{1}{2} M_{0} \alpha \sin \alpha(1+Z) \\
w=\frac{1}{2} M_{0}[\alpha \sin \alpha(1+Z)+2 \cos \alpha Z-2 Z]\end{array}$ \\
\hline Cycloid & $\cos \alpha$ & $\begin{array}{l}\xi=\frac{1}{3} M_{0} \sin \alpha(-2 \cos \alpha-1)+\frac{1}{3} Z M_{0} \sin \alpha(\cos \alpha-1) \\
\phi=\frac{1}{3} M_{0}\left(-\cos ^{2} \alpha+\cos \alpha-2\right)+\frac{1}{3} Z M_{0}\left(-2 \cos ^{2} \alpha+\cos \alpha+1\right) \\
w=\frac{1}{18} M_{0}\left(4 \cos ^{3} \alpha+3 \cos ^{2} \alpha-7\right)-\frac{1}{18} Z M_{0}\left(2 \cos ^{3} \alpha-3 \cos ^{2} \alpha+1\right)\end{array}$ \\
\hline Logarithmic spiral & $e^{\alpha}$ & $\begin{aligned} \xi= & \frac{1}{5} M_{0}\left(\sin \alpha+3 \cos \alpha-3 e^{\alpha} \cos \alpha-e^{\alpha} \sin \alpha\right) \\
& -\frac{1}{5} Z M_{0}\left(\sin \alpha+2 \cos \alpha-2 e^{\alpha} \cos \alpha+e^{\alpha} \sin \alpha\right) \\
\phi= & \frac{1}{5} M_{0}\left(\cos \alpha+3 \sin \alpha-e^{\alpha} \cos \alpha-2 e^{\alpha} \sin \alpha\right) \\
& +\frac{1}{5} Z M_{0}\left(\cos \alpha-2 \sin \alpha-e^{\alpha} \cos \alpha+3 e^{\alpha} \sin \alpha\right) \\
w= & \frac{1}{5} M_{0}\left(2 e^{\alpha} \cos \alpha+e^{\alpha} \sin \alpha-e^{2 \alpha} \cos \alpha-e^{2 \alpha} \sin \alpha-1\right) \\
& -\frac{1}{10} Z M_{0}\left(e^{\alpha} \cos \alpha+3 e^{\alpha} \sin \alpha-2 e^{\alpha} \cos \alpha+1\right)\end{aligned}$ \\
\hline Spiral & $2 \alpha$ & $\begin{aligned} \xi= & -\frac{1}{2} M_{0} \alpha(\sin \alpha+\alpha \cos \alpha)-\frac{1}{2} Z M_{0} \alpha(\sin \alpha+\alpha \cos \alpha) \\
\phi= & \frac{1}{2} M_{0}\left(-\alpha \cos \alpha+\sin \alpha-\alpha^{2} \sin \alpha\right) \\
& -\frac{1}{2} Z M_{0}\left(\alpha \cos \alpha-\sin \alpha-\alpha^{2} \sin \alpha\right) \\
w= & M_{0}\left(4 \cos \alpha+4 \alpha \sin \alpha-2 \alpha^{2} \cos \alpha-\alpha^{3} \sin \alpha-4\right) \\
& +Z M_{0}\left(-8 \cos \alpha-8 \alpha \sin \alpha+4 \alpha^{2} \cos \alpha+\alpha^{3} \sin \alpha+8\right)\end{aligned}$ \\
\hline
\end{tabular}

\subsection{A cantilever curved beam under a concentrated pure bending moment}

Consider a cantilever curved beam fixed at $\alpha=0$ and the free end $\alpha=\pi / 2$. A concentrated moment $M_{0}$ is applied at $\alpha=\pi / 2$ (Fig.4).

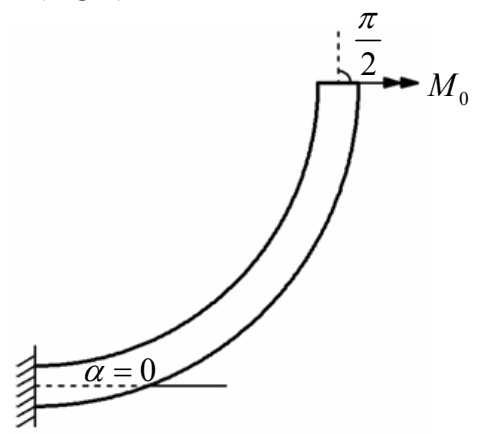

Fig.4 A cantilever curved beam under a concentrated moment $M_{0}$ at free end

Similarly as in the previous case, at fixed end $A_{3}=A_{4}=w_{0}=0$, and at the free end $Q=0$. The resultant moments at the free ends are $M_{u}(\pi / 2)=M_{0}, M_{T}(\pi / 2)=0$. The conditions yield

$$
M_{u}=M_{0} \cos \alpha, M_{T}=-M_{0} \sin \alpha .
$$

Substituting Eqs.(30) into Eqs.(15,16), after some calculation, the rotation angles $\phi, \xi$ are 


$$
\begin{aligned}
& \phi=M_{0}\left\{-\cos \alpha R_{s s}+\sin \alpha R_{s c}+Z\left(\cos \alpha R_{c c}+\sin \alpha R_{s s}\right)\right\}, \\
& \xi=M_{0}\left\{\sin \alpha R_{s s}+\cos \alpha R_{s c}+Z\left(-\sin \alpha R_{c c}+\cos \alpha R_{s c}\right)\right\} .
\end{aligned}
$$

The deflection $w$ can be found by

$$
w=M_{0} \int_{0}^{\alpha}\left\{\sin \alpha R_{s s}+\cos \alpha R_{s c}+Z\left(-\sin \alpha R_{c c}+\cos \alpha R_{s c}\right)\right\} R d \alpha .
$$

Again there is no simple form of $w$. Once the radius of curvature is assigned, the geometric quantities can be evaluated. Then the deformation can be expressed in terms of $\alpha$. Table 2 lists some analytical solutions of various curved beams such as circle, cycloid, logarithmic spiral, and spiral curved beams.

So far the general solutions are expressed in terms of the geometrical quantities. In curved beam analysis, the moments of curve with respect to $x, y$ axes play important roles.

\begin{tabular}{|c|c|c|}
\hline Curve Type & $R$ & $\xi, \phi, w$ \\
\hline Circle & 1 & $\begin{array}{l}\xi=-\frac{1}{2} M_{0} \alpha \sin \alpha(1+Z) \\
\phi=\frac{1}{2} M_{0}[\alpha \cos \alpha(1+Z)+\sin \alpha(-1+Z)] \\
w=\frac{1}{6} M_{0}[\sin \alpha(1-Z)-2 \sin \alpha(2+Z)+3 \alpha \cos \alpha(1+Z)]\end{array}$ \\
\hline Cycloid & $\cos \alpha$ & $\begin{array}{l}\xi=\frac{1}{3} M_{0}\left(-2 \cos ^{2} \alpha+\cos \alpha+1\right)+\frac{1}{3} Z M_{0}\left(2 \cos ^{4} \alpha+\cos ^{2} \alpha-\cos \alpha-2\right) \\
\phi=\frac{1}{3} M_{0} \sin \alpha\left(-2 \cos ^{3} \alpha+2 \cos \alpha+1\right)+\frac{1}{3} Z M_{0} \sin \alpha(2 \cos \alpha+1) \\
w=\frac{1}{6} M_{0}(\sin \alpha+\sin \alpha \cos \alpha+\alpha)+\frac{1}{6} Z M_{0}\left(\frac{1}{20} \sin 5 \alpha+\frac{7}{12} \sin 3 \alpha-\frac{1}{2} \sin 2 \alpha-1\right)\end{array}$ \\
\hline $\begin{array}{l}\text { Logarithmic } \\
\text { spiral }\end{array}$ & $e^{\alpha}$ & $\begin{aligned} \xi= & \frac{1}{5} M_{0}\left(\cos \alpha+2 \sin \alpha-e^{\alpha} \cos \alpha+3 e^{\alpha} \sin \alpha\right) \\
& +\frac{1}{5} Z M_{0}\left(\cos \alpha+3 \sin \alpha-e^{\alpha} \cos \alpha-2 e^{\alpha} \sin \alpha\right) \\
\phi= & \frac{1}{10} M_{0}\left(-4 \cos \alpha+2 \sin \alpha+5 e^{\alpha} \cos \alpha-e^{\alpha} \cos 3 \alpha-2 e^{\alpha} \sin 3 \alpha\right) \\
& +\frac{1}{5} Z M_{0}\left(-3 \cos \alpha+\sin \alpha+3 e^{\alpha} \cos \alpha+e^{\alpha} \sin \alpha\right) \\
w= & \frac{1}{10} M_{0}\left(3 e^{\alpha} \cos \alpha-e^{\alpha} \sin \alpha-2 e^{2 \alpha} \cos \alpha+2 e^{2 \alpha} \sin \alpha-1\right) \\
& +\frac{1}{5} Z M_{0}\left(-e^{\alpha} \cos \alpha+2 e^{\alpha} \sin \alpha-e^{2 \alpha} \sin \alpha+1\right)\end{aligned}$ \\
\hline Spiral & $2 \alpha$ & $\begin{aligned} \xi= & \frac{1}{2} M_{0}\left(\sin \alpha-\alpha \cos \alpha+\alpha^{2} \sin \alpha\right) \\
& +\frac{1}{2} Z M_{0}\left(\sin \alpha-\alpha \cos \alpha-\alpha^{2} \sin \alpha\right) \\
\phi= & \frac{1}{2} M_{0}\left(-4 \sin \alpha \cos ^{2} \alpha+\alpha \sin \alpha+2 \cos \alpha+\alpha^{2} \cos \alpha-2 \cos ^{3} \alpha\right) \\
& +\frac{1}{2} Z M_{0} \alpha \sin \alpha(\alpha+1) \\
w= & M_{0}\left(-3 \sin \alpha+3 \alpha \sin \alpha+2 \alpha^{2} \sin \alpha-\alpha^{3} \cos \alpha\right) \\
& +Z M_{0}\left(9 \sin \alpha-9 \alpha \sin \alpha-4 \alpha^{2} \sin \alpha+\alpha^{3} \cos \alpha\right)\end{aligned}$ \\
\hline
\end{tabular}

Table 2 Dimensionless deflections of various curved cantilever beams under a concentrated moment $M_{0}$ at free end 


\subsection{A cantilever curved beam under a concentrated load at free end}

Consider a cantilever curved beam with variable curvature, fixed at $\alpha=0$, and free at $\alpha=\pi / 2$ under a concentrated load $P$ at $\alpha=0$ (Fig.5).

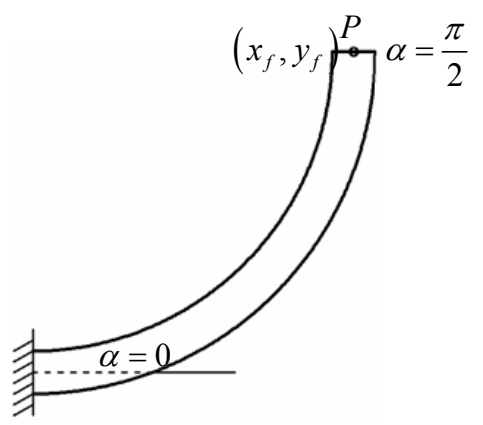

Fig.5 A cantilever curved beam under a concentrated load $P$

Assume that the coordinates at the free end are $\left(x_{f}, y_{f}\right)$. From Eq.(9), the shear force can be calculated

$$
Q=-P \text {. }
$$

Substituting Eq.(33) into Eqs.(11,12), the moments yield

$$
\begin{aligned}
& M_{T}=A_{1} \cos \alpha+A_{2} \sin \alpha+P y \cos \alpha-P y \sin \alpha, \\
& M_{u}=-A_{1} \sin \alpha+A_{2} \cos \alpha-P y \sin \alpha-P y \cos \alpha .
\end{aligned}
$$

Due to the free end conditions $M_{T}(\pi / 2)=0, M_{u}(\pi / 2)=0$, the constants $A_{1}$ and $A_{2}$ are

$$
A_{1}=P y_{f}, \quad A_{2}=-P x_{f} .
$$

Substituting Eqs. $(34,35)$ into Eqs. $(14,15)$, the rotation angles can be evaluated

$$
\begin{aligned}
\phi & =P \cos \alpha\left(y_{f} R_{s s}-x_{f} R_{s c}-\int_{0}^{\alpha} y R \sin \alpha \sin \alpha d \alpha-\int_{0}^{\alpha} x R \sin \alpha \cos \alpha d \alpha\right) \\
& +P \sin \alpha\left(-y_{f} R_{s c}+x_{f} R_{c c}+\int_{0}^{\alpha} y R \sin \alpha \cos \alpha d \alpha+\int_{0}^{\alpha} x R \cos \alpha \cos \alpha d \alpha\right) \\
& +Z P \cos \alpha\left(y_{f} R_{c c}+x_{f} R_{s c}-\int_{0}^{\alpha} y R \cos \alpha \cos \alpha d \alpha+\int_{0}^{\alpha} x R \sin \alpha \cos \alpha d \alpha\right) \\
& +Z P \sin \alpha\left(y_{f} R_{s c}+x_{f} R_{s s}-\int_{0}^{\alpha} y R \sin \alpha \cos \alpha d \alpha+\int_{0}^{\alpha} x R \sin \alpha \sin \alpha d \alpha\right) \\
\xi & =P \sin \alpha\left(-y_{f} R_{s s}+x_{f} R_{s c}+\int_{0}^{\alpha} y R \sin \alpha \sin \alpha d \alpha+\int_{0}^{\alpha} x R \sin \alpha \cos \alpha d \alpha\right) \\
& +P \cos \alpha\left(-y_{f} R_{s c}+x_{f} R_{c c}+\int_{0}^{\alpha} y R \sin \alpha \cos \alpha d \alpha+\int_{0}^{\alpha} x R \cos \alpha \cos \alpha d \alpha\right) \\
& -Z P \sin \alpha\left(y_{f} R_{c c}+x_{f} R_{s c}-\int_{0}^{\alpha} y R \cos \alpha \cos \alpha d \alpha+\int_{0}^{\alpha} x R \sin \alpha \cos \alpha d \alpha\right) \\
& +Z P \cos \alpha\left(y_{f} R_{s c}+x_{f} R_{s s}-\int_{0}^{\alpha} y R \sin \alpha \cos \alpha d \alpha+\int_{0}^{\alpha} x R \sin \alpha \sin \alpha d \alpha\right) .
\end{aligned}
$$

The deformation $w$ can be obtained by integrating Eq.(36b) with respect to arc length, it is

$$
w=\int_{0}^{\alpha} \xi R d \alpha+w_{0} .
$$

Some analytical solutions of circle, cycloid, logarithmic spiral, and spiral, curved beams are shown in Table 3. 
Table 3 Dimensionless deflections of various curved cantilever beams under a concentrated load $P$ at free end

\begin{tabular}{|c|c|c|}
\hline $\begin{array}{c}\text { Curve } \\
\text { Type }\end{array}$ & $R$ & $\xi, \phi, w$ \\
\hline Circle & 1 & $\begin{aligned} \xi=\frac{1}{2} P[ & \left.2 Z(1-\cos \alpha)+(1+Z)\left(x_{f}+y_{f}-1\right) \alpha \sin \alpha+P(1-Z) x_{f} \sin \alpha\right] \\
\phi=\frac{1}{2} P[ & (1+Z)(\cos \alpha-\sin \alpha)+(1-Z) y_{f} \sin \alpha \\
& \left.\quad-(1+Z) y_{f} \alpha \cos \alpha+(1+Z) x_{f} \alpha \sin \alpha\right] \\
w=\frac{1}{6} P[ & 4(1+2 Z) \sin \alpha-3(1+Z) x_{f} \alpha \sin \alpha-2(2+Z) y_{f} \sin \alpha \\
& \quad-6 Z x_{f}(1-\cos \alpha)-12 Z \alpha+(1-Z)\left(y_{f}-1\right) \sin \alpha \cos ^{2} \alpha \\
& \left.+(1-Z)\left(y_{f}-1\right) \sin ^{3} \alpha+3(1+Z)\left(y_{f}-1\right) \alpha \cos \alpha\right]\end{aligned}$ \\
\hline Cycloid & $\cos \alpha$ & $\begin{aligned} \xi=\frac{1}{18} P[ & 6(1-Z) x_{f} \sin \alpha+3(2+Z)\left(2 x_{f}-\alpha\right) \sin \alpha \cos \alpha \\
& +6(1+2 Z) y_{f}-6(2+Z) y_{f} \cos ^{2} \alpha+4(1-Z) \cos \alpha \\
& +6(1-Z) b \cos \alpha+(2 Z+\cos \alpha) \cos \alpha+2 Z-5] \\
\phi=\frac{-1}{18} P[ & 6(1+2 Z) y_{f} \sin \alpha \cos \alpha+(1+5 Z) \sin \alpha \cos \alpha \\
& +3(2+Z)\left(\alpha-2 x_{f}\right)-3(1+2 Z)\left(\alpha-2 x_{f}\right) \cos ^{2} \alpha \\
& \left.+6(1-Z) x_{f} \cos \alpha-2(2+Z) x_{f} \sin \alpha\right] \\
w=\frac{-1}{1350} P & {\left[75(7-Z) x_{f}+75(2+Z)(\alpha \sin \alpha \cos \alpha)-22(15+7) \sin \alpha\right.} \\
& +30(5 Z-4) y_{f} \sin \alpha \cos ^{2} \alpha+225(1-Z) y_{f} \sin \alpha \cos \alpha \\
& -9(Z-5) \sin ^{5} \alpha+5(9 Z-10) \sin { }^{3} \alpha+225(1-Z) y_{f} \alpha \\
& -(165+62 Z) \sin \alpha \cos ^{2} \alpha+9\left(5-Z-10 y_{f}\right) \sin \alpha \cos ^{4} \alpha \\
& +60(5+Z) x_{f} \cos ^{3} \alpha+225(Z-1) x_{f} \cos ^{2} \alpha-90 Z a \\
& +45 Z \alpha \cos ^{2} \alpha\left(\cos ^{2} \alpha-\sin ^{2} \alpha\right)+30\left(5 \cos ^{2} \alpha+Z\right) \alpha \sin \alpha \\
& +30\left(3 \sin ^{2} \alpha+10 Z\right) y_{f} \sin ^{3} \alpha+60(5 Z-4) y_{f} \sin \alpha \\
& \left.+15 Z \alpha \sin ^{2} \alpha \cos ^{2} \alpha\right]\end{aligned}$ \\
\hline
\end{tabular}

The results of deflection at $\alpha=\pi / 2$ shown in Table 4 are also compared with the results by ANSYS and by Castigliano's method. The results are all consistent. In ANSYS analysis, the Poisson's ratio is included.

Table 4.1 The rotation deformation $\xi$ at $\alpha=\pi / 2$ of a circular beam with Young's modulus $\mathrm{E}=2 \times 10^{11}$, Poisson's ratio $\nu=0.3$, and $R_{0}=10$.

\begin{tabular}{cccc}
\multicolumn{4}{c}{$\mathrm{E}=2 \times 10.3$, and $R_{0}=10}$. \\
\hline$\xi$ & Present & Castigliano & ANSYS \\
\hline Error $(\%)$ & $0.6927710840 \times 10^{-8}$ & $0.6927710840 \times 10^{-8}$ & $0.68473 \times 10^{-8}$ \\
\hline & & 0 & $1.16071 \%$
\end{tabular}

Table 4.2 The rotation deformation $\phi$ at $\alpha=\pi / 2$ of a circular beam with Young's modulus $\mathrm{E}=2 \times 10^{11}$, Poisson's ratio $v=0.3$, and $R_{0}=10$.

\begin{tabular}{cccc}
\hline & Present & Castigliano & ANSYS \\
\hline$\phi$ & $0.3050697440 \times 10^{-8}$ & $0.3050697440 \times 10^{-8}$ & $0.3050697440 \times 10^{-8}$ \\
\hline Error $(\%)$ & & 0 & $1.70116 \%$
\end{tabular}


Table 4.3 The displacement $w$ of a circular beam with Young's modulus $E=2 \times 10^{11}$, Poisson's ratio $v=0.3$, and $R_{0}=10$.

\begin{tabular}{cccc}
\hline & Present & Castigliano & ANSYS \\
\hline$w$ & $-0.73775 \mathrm{E} \times 10^{-7}$ & $-0.73775 \times 10^{-7}$ & $-0.752078917 \times 10^{-7}$ \\
\hline Error $(\%)$ & & 0 & $1.90524 \%$
\end{tabular}

\section{Out of plane stability Analysis}

To investigate the stability, the in plane forces $N, V$ must be included. The analytical solutions become very limited because of the coupled system of in plane and out of plane terms. Here only analytical solutions are interested.

Consider a circular ring under a uniform distributed pressure $q$ (Fig.6).

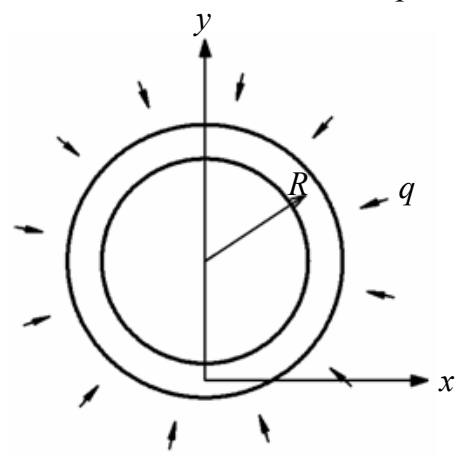

Fig.6 A circle ring under a uniform pressure $q$

The in plane analysis has been investigated by some researches. However the out of plane stability of the ring under uniform pressure is absent as far as authors' concern.

By changing variable Eq.(8), the in plane resultants Eqs.(2) become

$$
\frac{d N}{d \alpha}+V=0, \quad-N+\frac{d V}{d \alpha}-q R=0 .
$$

The solutions of $N$ and $V$ are

$$
N=A_{1} \cos \alpha+A_{2} \sin \alpha-q R, \quad V=A_{1} \sin \alpha-A_{2} \cos \alpha .
$$

Due to symmetry, the reaction conditions are given by $N(0)=-q R, N(\pi / 2)=-q R$. The conditions yield

$$
A_{1}=A_{2}=0
$$

or

$$
N=-q R, \quad V=0 .
$$

Since there is no out of plane distributed load, the shear force $Q$ by Eq.(3c) yields

$$
Q=0 \text {. }
$$

By using changing of variable Eq.(8), the moments in Eqs.(3) are

$$
M_{u}+\frac{d M_{T}}{d \alpha}=0, \quad \frac{d M_{u}}{d \alpha}-M_{T}+q R^{2} \xi=0 .
$$

There are several cases which can be discussed.

\subsection{In the case of $C \rightarrow \infty$}

This is the case that torsion rigidity is much bigger than flexural rigidity, so that $\frac{d \phi}{d \alpha}=\xi$ 
Therefore

$$
M_{u}=\frac{d \xi}{d \alpha}+\phi=\frac{d^{2} \phi}{d \alpha^{2}}+\phi
$$

Substituting Eq.(44) into Eqs.(42) and taking derivative once, Eq.(42) can be simplified to

$$
\frac{d^{4} \phi}{d \alpha^{4}}+2 \lambda^{2} \frac{d^{2} \phi}{d \alpha^{2}}+\phi=0
$$

where

$$
2 \lambda^{2}=\frac{q R^{3}}{E I}+2
$$

The solution of Eq.(45) is expressed as

$$
\phi=B_{1} \sin \beta_{1} \alpha+B_{2} \cos \beta_{1} \alpha+B_{3} \sin \beta_{2} \alpha+B_{4} \cos \beta_{2} \alpha,
$$

where

$$
\beta_{1}^{2}=\lambda^{2}+\sqrt{\lambda^{4}-1}, \quad \beta_{2}^{2}=\lambda^{2}-\sqrt{\lambda^{4}-1} .
$$

Assuming the first mode being symmetric, since the displacement $w$ is even, the rotation angle $\xi$ which is the derivative of $w$ is an odd function. Hence $\phi$ is an even function. Therefore only keep the even function terms in Eq.(47),

$$
\phi=B_{2} \cos \beta_{1} \alpha+B_{4} \cos \beta_{2} \alpha .
$$

The rotation angle $\xi$ is by using Eq.(43)

$$
\xi=-\beta_{1} B_{2} \sin \beta_{1} \alpha-\beta_{2} B_{4} \cos \beta_{2} \alpha .
$$

Integrating Eq.(50) once, the displacement $w$ is

$$
w=w_{0}+R\left(B_{2} \cos \beta_{1} \alpha+B_{4} \cos \beta_{2} \alpha\right) .
$$

The stability analysis then can be investigated by implying suitable boundary conditions. Due to symmetry, choose half circle $-\pi / 2 \leqq \alpha \leqq \pi / 2$. At two ends $\alpha=-\pi / 2$, $\alpha=\pi / 2$, the in plane rotation $\varphi$ vanishes due to symmetry. Only horizontal displacements $u, v$ occur. At $\alpha=0$ and $\alpha=\pi$, they are symmetric points such that $w(0)=w(\pi)=0$. Due to symmetry, the rotation $\xi$ at $\alpha=0, \alpha=\pi / 2, \alpha=\pi$ are all vanished. Hence $\xi(0)=\xi(\pi / 2)=\xi(\pi)=0$. Apply the conditions of displacement of $w$, it implies that

$$
R\left(B_{2} \cos \beta_{1} \pi+B_{4} \cos \beta_{2} \pi\right)=0, \quad-B_{2} \beta_{1} \sin \beta_{1} \frac{\pi}{2}-B_{4} \beta_{2} \sin \beta_{2} \frac{\pi}{2}=0 .
$$

With the help of boundary conditions $\xi(\pi / 2)=\xi(\pi)=0$, the nontrivial solution of Eqs.(52) yields

$$
2 \sin \frac{\beta_{1} \pi}{2} \sin \frac{\beta_{2} \pi}{2}\left(\cos \frac{\beta_{1} \pi}{2}-\cos \frac{\beta_{2} \pi}{2}\right)=0 .
$$

The possible solutions of the first mode are $\beta_{1}=2, \beta_{2}=2$ or $\beta_{1}-\beta_{2}= \pm 2$. The critical loads of each case are

$$
q_{c r}=\frac{9}{4} \frac{E I}{R^{3}} .
$$

or

$$
q_{c r}=16 \frac{E I}{R^{3}} .
$$

Hence the dominated critical of the symmetric mode is

$$
q_{c r}=\frac{9}{4} \frac{E I}{R^{3}} .
$$

It is also possible to find an anti-symmetric mode. For instance it is assumed that $w(\alpha)=-w(-\alpha), \xi(\alpha)=\xi(-\alpha)$. However the nontrivial solution does not exist. Hence there is 
no such a mode.

There are some choices of the boundary conditions along the out-of-plane directions. Some suitable boundary conditions can also be assigned. For instance consider a torsion mode. Assume the torsion mode is symmetric with respect to $y$-axis. Then no twist angle occurs at $\alpha=0, \alpha=\pi, \alpha= \pm \pi / 2$, i.e.

$$
\phi(0)=0, \quad \phi\left( \pm \frac{\pi}{2}\right)=0
$$

Substituting Eq.(56) into Eq.(47), the characteristic equation is

$$
\cos \beta_{1} \frac{\pi}{2}-\cos \beta_{2} \frac{\pi}{2}=0,
$$

or

$$
2 \sin \frac{\pi\left(\beta_{1}+\beta_{2}\right)}{4} \sin \frac{\pi\left(\beta_{1}-\beta_{2}\right)}{4}=0 .
$$

Hence the possible solutions are

$$
\beta_{1}+\beta_{2}=4, \quad \beta_{1}-\beta_{2}=4 .
$$

Substituting Eqs.(58) into Eq.(48), the critical load can be obtained. Choose the small one,

$$
q_{c r}=2 \frac{E I}{R^{3}} \text {. }
$$

\subsection{In the limited case $E I \rightarrow \infty$}

There is almost no flexural rotation. Hence

$$
\frac{d \xi}{d \alpha}=-\phi
$$

Substituting Eqs.(60) into Eq.(5b), the torque can be expressed as

$$
M_{T}=\frac{C}{R}\left(-\frac{d^{2} \xi}{d \alpha^{2}}-\xi\right) .
$$

The force balance Eqs.(42) can then be simplified to

$$
-\frac{d^{2} M_{T}}{d \alpha^{2}}+M_{T}+q R^{2} \xi=0 .
$$

Substituting Eqs.(61) into Eq.(62), it yields

$$
\frac{d^{4} \xi}{d \alpha^{4}}+2 \frac{d^{2} \xi}{d \alpha^{2}}+\xi+\lambda^{2} \xi=0
$$

where

$$
\lambda^{2}=\frac{1}{Z} \frac{q R^{3}}{E I} .
$$

The nontrivial solution does not exist in this case.

\section{Conclusion}

The static analysis of curved beam of variable curvature on 2-D plane is derived. The general solutions under various loadings are demonstrated. The solutions are expressed in terms of angle of tangent. The first and second moments of arc length with respect to $x, y$ axes are defined as fundamental geometric quantities to express the general solutions. The analytical solutions of circular, cycloid, and spiral curved beams under pure torque and under pure bending are demonstrated. The analytical solutions of circle, cycloid logarithmic spiral and spiral cantilever curved beams under concentrated load are presented as well. The out-of-plane critical load of a circular ring under uniform radial load is derived. Various out-of-plane boundary conditions are considered. Several critical loads can be found based on various boundary conditions. The results are compared to the result by ANSYS. The results are consistent. 


\section{References}

[1] Green A.E., Naghdi P.M., Wenner M.L., On the theory of rods I. Derivations from the three-dimensional equations, Proc. Roy. Soc. Lond. A. 337, 1974, 451-483.

[2] Green A.E., Naghdi P.M., Wenner M.L., On the theory of rods II. Developments by direct approach, Proc. Roy. Soc. Lond. A. 337, 1974, 485-507.

[3] Naghdi P.M., Finite deformation of elastic rods and shells, Proc. IutamSymp. On Finite Elasticity, LeihighUniv., Aug. 1980, 47-103.

[4] Green A.E., Naghdi P.M., Non-isothermal theory of rods, plates and shells. Int. J. Solids Strucs. 6, 1970, 209-244.

[5] Green A.E., Laws N., Remarks on the theory of rods, J. Elasticity, 3, 1973, 179-184.

[6] Yu A., Fang M., Ma X., Theoretical research on natural curved and twisted beam under complicated loads, Computer Structure, 2002, 80, 2529-2536.

[7] Malekzadeh P., Karami G., Out-of-plane static analysis of circular arches by DQM, Int. J. Solid Structure, 2003, 40, 6527-6545.

[8] Kang K., Bert C.W., Striz A.G., Static analysis of a circular shaft subjected to end torques, Int. J. Solid Structure, 1996, 33, 11, 1587-1596.

[9] Yildirim V., Common effect of rotary inertia and shear deformation on the out-of-plane natural frequencies of composite circular bars, Composites part B: 32, 2001, 687-695.

[10] Huang C.S., Tseng Y.P., Chang S.H., Out-of-plane responses of noncircular curved beams by numerical Laplace transform, J. Sound Vibration, 1998, 215, 3, 407-424.

[11] Rhodes J., A semi-analytical approach to buckling analysis for composite structures, Composite Structures, 1996, 35, 93-99.

[12] Attard M.M., Minh N.G., Foster S.J., Finite element analysis of out-of-plane buckling of reinforced concrete walls, Computer Structures, 1996, 61, 6, 1037-1042.

[13] Lim N.H., Kang Y.J., Out of plane stability of circular arches, Int. J. Mechanical Science, 2004, 46, 1115-1137.

[14] Lin K.C., Hsieh C.M., The closed form general solutions of 2-D curved laminated beams of variable curvatures, Composite Structures, 79, 2007, 606-618. 